\title{
Implantable vagus nerve stimulation system performance is not affected by internal or external defibrillation shocks
}

\author{
Imad Libbus $^{1}$ (1) $\cdot$ Scott R. Stubbs ${ }^{1} \cdot$ Scott T. Mazar ${ }^{1} \cdot$ Scott Mindrebo $^{1} \cdot$ Bruce H. KenKnight $^{1} \cdot$ Lorenzo A. DiCarlo $^{1}$
}

Received: 12 May 2021 / Accepted: 9 August 2021 / Published online: 31 August 2021

(C) The Author(s) 2021

\begin{abstract}
Purpose Autonomic regulation therapy (ART) for heart failure (HF) is delivered using vagus nerve stimulation (VNS), and has been associated with improvement in cardiac function and HF symptoms. VNS is delivered using an implantable pulse generator (IPG) and a lead placed around the cervical vagus nerve. Because HF patients may receive concomitant cardiac defibrillation therapy, testing was conducted to determine the effect of defibrillation (DF) on VNS system performance.

Methods Normal swine $(n=4)$ with VNS system implants on the right cervical vagus nerve received sequential defibrillation shocks with three defibrillation systems: an implantable cardioverter defibrillator (ICD), a subcutaneous ICD (S-ICD), and an external cardioverter defibrillator (ECD). Each system delivered a series of bipolar high-energy shocks and reversepolarity high-energy shocks.

Results The specified cardiac defibrillation shocks were delivered successfully from each of the three defibrillation systems to all animals. After each shock series, interrogation of the IPG confirmed that software and data were unchanged from preprogrammed values. After all of the defibrillation shocks were delivered, the IPGs underwent and passed comprehensive electrical testing demonstrating proper system function. No shifts in IPG parameters or ART system failures were observed, and histologic evaluation of the vagus nerve revealed no anatomic changes.

Conclusions Implantable VNS systems were tested in vivo for immunity to defibrillation via ICD, S-ICD, and ECD, and were found to be unaffected by a series of high-energy defibrillation shocks. These results confirm that ART systems are capable of continuing to function after defibrillation and the cervical vagus nerve is anatomically unaffected.
\end{abstract}

Keywords Autonomic regulation therapy $\cdot$ Vagus nerve $\cdot$ Vagus nerve stimulation $\cdot$ Heart failure $\cdot$ Implantable cardioverter defibrillator · Defibrillation · Autonomic nervous system

\section{Introduction}

Heart failure (HF) is characterized by hemodynamic abnormalities that are associated with a marked autonomic imbalance consisting of increased sympathetic activity and withdrawal of parasympathetic tone. This pathological adrenergic hyperactivation contributes to the progression of $\mathrm{HF}$ and increases the risk of mortality and morbidity independent of left ventricular ejection fraction (EF) and ventricular arrhythmias [1].

Autonomic regulation therapy (ART) is a novel therapy for the management of HF. ART uses cervical vagus nerve

Imad Libbus

Imad.Libbus@livanova.com

1 LivaNova USA, Inc., Houston, TX, USA stimulation (VNS) to increase parasympathetic activity and to restore autonomic balance. ART is delivered using chronic stimulation through an electrical lead that is implanted around the cervical vagus nerve without requiring any mapping for placement [2]. An ART system consists of a self-sizing, atraumatic helical lead that is placed around the cervical vagus nerve without requiring any mapping for placement and has a low rate of complications and failures [3]. The lead is connected to an implantable pulse generator (IPG) that is implanted in an infraclavicular subcutaneous pocket and delivers neurostimulation according to programmed stimulation parameters (current amplitude, pulse width, frequency, and duty cycle) that can be adjusted wirelessly by inductive telemetry using a handheld wand and programming computer (Fig. 1).

In the ANTHEM-HF study, ART delivered using openloop VNS was associated with long-term improvement in left ventricular function, 6-min walk distance, NYHA class, 


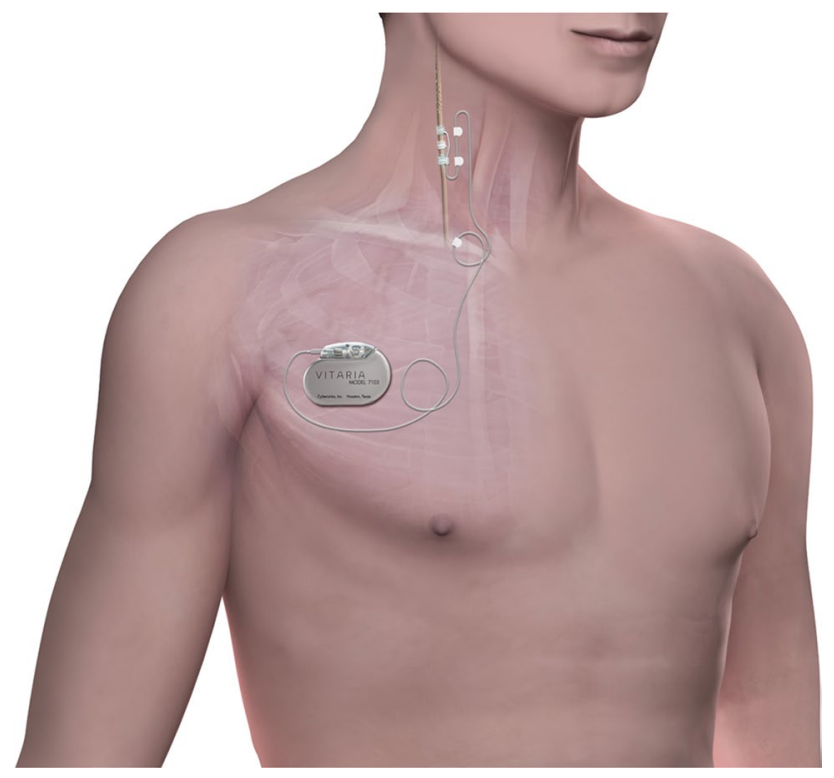

Fig. 1 Autonomic regulation therapy (ART) system consisting of an implantable pulse generator and a lead that is placed around the right cervical vagus nerve

heart rate, heart rate variability, and quality of life in patients with HF and reduced EF (HFrEF) [4-6]. The impact of ART on mortality and morbidity is being evaluated in an ongoing pivotal study in patients with HFrEF [7].

International guidelines for the treatment of HF recommend implantable cardioverter defibrillator (ICD) therapy for patients with HFrEF and at risk for ventricular arrhythmias [8-11]. Because HFrEF patients may be recipients of both ART and ICD and exposed to either external or internal high-energy defibrillation, it is important to establish whether defibrillation shocks at industry-maximum levels may have an effect on the operation of ART systems. ART systems are designed with high voltage protection, and previous benchtop testing according to the international standard for determining defibrillation immunity of implantable medical devices (ISO 14,708-1) [12] established that implantable VNS systems were unaffected by high-energy defibrillation in vitro [13].

The purpose of this study was to determine whether functional performance of ART systems is detrimentally affected by internal and external defibrillation in vivo using defibrillation shocks delivered by an ICD, a subcutaneous ICD (S-ICD), and an external cardioverter defibrillator (ECD).

\section{Methods}

The study was approved by the American Preclinical Services Institutional Animal Care and Use Committee (IACUC) and conformed to the National Institutes of Health Guide for the Care and Use of Laboratory Animals. Normal Yorkshire swine ( $n=4)$ weighing 40 to $50 \mathrm{~kg}$ were implanted with the VITARIA® ART system (LivaNova USA, Houston, TX; Fig. 1), consisting of a Model 7304 lead that was placed around the right cervical vagus nerve and a Model 7103 implantable pulse generator (IPG) that was placed in a subcutaneous pocket in the animal's right dorsolateral neck area, as previously described [14]. All procedures were performed under general anesthesia and sterile conditions.

After implantation, the IPG was activated and programmed to deliver chronic vagus nerve stimulation using $2.5 \mathrm{~mA}$ amplitude, $250 \mu \mathrm{sec}$ pulse width, $5 \mathrm{~Hz}$ frequency, and a duty cycle of $14 \mathrm{~s}$ on/66 s off; these parameter values are typical in clinical setting.

For each of the four animals, the following three defibrillation systems were sequentially implanted, used, and then explanted prior to the implantation of the next system. The placement of the implants is shown in Fig. 2.

1. ICD (Spring Quattro Secure S Ventricular Lead DF4, Medtronic, Minneapolis, MN). The ICD lead was implanted transvenously into the right ventricular apex under fluoroscopic guidance and connected to an external pulse generator, which delivered defibrillation shocks at $50 \mathrm{~J}$.

2. S-ICD (Emblem S-ICD, Boston Scientific, Saint Paul, $\mathrm{MN})$. The lead was implanted subcutaneously in the standard L-shaped configuration [15]. A stainless steel disk the size of the S-ICD IPG (70 mm diameter, $13 \mathrm{~mm}$ thick) was implanted in the left thoracic region and used as a return electrode. Primary (coil A) and secondary (coil B) defibrillation vectors were sequentially tested. The electrodes were connected to an external pulse generator, which delivered defibrillation shocks at $75 \mathrm{~J}$.

3. ECD (M-Series Biphasic Defibrillator, Zoll Medical, Boston, MA). Defibrillation pads $\left(40 / 50 \mathrm{~cm}^{2}\right)$ were placed on the chest in the conventional anterior configuration (right pectoral to left thoracic), and defibrillation shocks were delivered at $200 \mathrm{~J}$.

The order in which the three defibrillation systems (ICD, S-ICD, and ECD) were used was randomly selected for each animal using a six-sided die to select among the six possible options. In each configuration, a series of high voltage biphasic defibrillation pulses were delivered: three pulses separated by $20 \mathrm{~s}$, followed by a 60 -s pause, followed by three pulses separated by $20 \mathrm{~s}$ with reverse polarity. The application of the defibrillation pulses was not synchronized to the output of the VITARIA IPG.

Each IPG was interrogated and the programmed device parameters were compared before and after each set of shocks. After all of the planned shock applications were delivered, each IPG underwent evaluation for damage using electrical testing that duplicated what is performed at the end of the manufacturing process for ART systems to confirm 


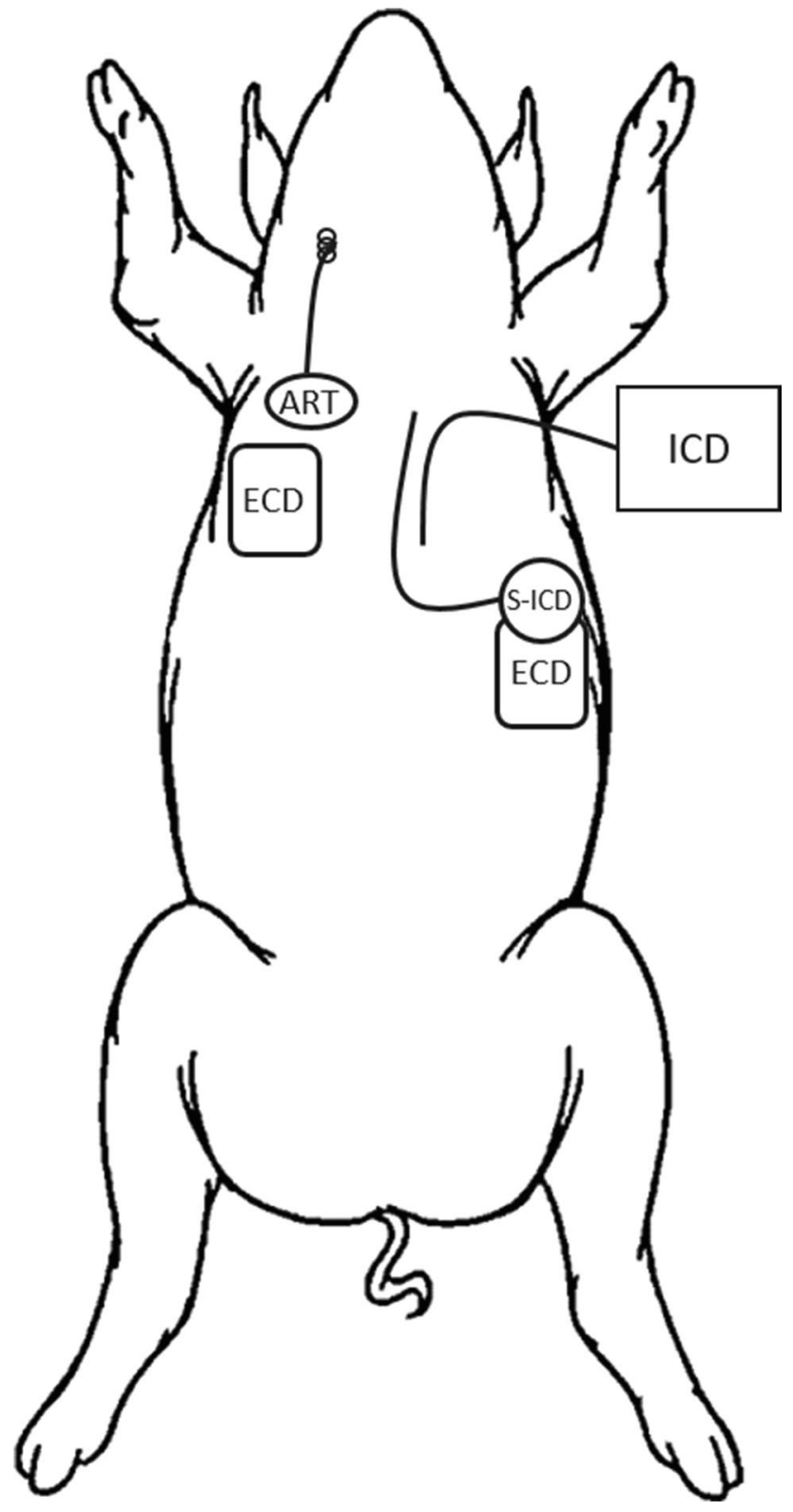

Fig. 2 Placement of the ART system and the three defibrillation systems. Each of the defibrillation systems was sequentially implanted, used, and then explanted prior to the implantation of the next system

that each ART system met all of the required functional specifications, as previously described [16].

To confirm that autonomic function was intact, acute heart rate dynamics were assessed in response to vagus nerve stimulation, as previously described [17]. VNS intensity was sequentially raised from 0 to $3.0 \mathrm{~mA}$ in $1 \mathrm{~mA}$ increments, and acute heart rate change derived from the ECG was measured. This assessment was performed before and after the series of defibrillation shocks.

At the end of the procedure, the right and left cervical vagus nerves were removed for histology assessment. The
Table 1 Randomized order of defibrillation application

\begin{tabular}{llll}
\hline $\begin{array}{l}\text { Animal } \\
\text { number }\end{array}$ & 1st defibrillation & 2nd defibrillation & 3rd defibrillation \\
\hline 1 & S-ICD & ECD & ICD \\
2 & ICD & ECD & S-ICD \\
3 & S-ICD & ICD & ECD \\
4 & ECD & ICD & S-ICD \\
\hline
\end{tabular}

entire nerves were harvested from the thoracic inlet to the upper neck, immersion fixed in 10\% neutral buffered formalin, and embedded in paraffin. Right cervical nerve sections were taken at sites proximal to the electrode, three sections underneath the electrodes, and distal to the electrode. Nerves sections were stained with hematoxylin and eosin (H\&E) and Luxol fast blue (LFB) and evaluated by an independent study pathologist.

\section{Results}

The order in which the three defibrillation systems were randomized in each animal is shown in Table 1. All defibrillation tests were performed successfully in all animals, with no deviations from the protocol.

In two of the animals, a defibrillation shock induced ventricular fibrillation, and an additional external defibrillation shock was required to terminate the arrhythmia. In total, each animal implanted with the VITARIA system received a total of 24-26 high-energy shocks through either an ICD, S-ICD, or ECD configuration.

After each set of shocks, each IPG was interrogated, and the interrogated data (serial number, patient ID, output current, frequency, pulse width, on-time, and off-time) was found to be unchanged from the programmed values prior to the shocks. Comprehensive electrical testing of each ART system after delivery of the final shock confirmed no device failures or parameter shifts and that they were still within the manufacturer's functional specifications. The output of the pulse generator was measured and found to be within the manufacturing tolerance for each programmed stimulation parameter.

An intensity-dependent heart rate response was observed before and after the series of defibrillation pulses, indicating the presence of autonomic engagement. The application of repeated shocks altered the autonomic conditions, resulting in an attenuated response post-defibrillation (from a peak response of $5.5 \pm 3.1 \mathrm{bpm}$ to a peak response of $2.1 \pm 6.4 \mathrm{bpm}$ ).

Histological analysis with H\&E and LFB of the left and right cervical vagus nerves confirmed the absence of any damage to the nerve (Fig. 3). The nerves from all four animals showed no necrosis and minimal inflammation and hemorrhage. There was no minimal evidence of thermal injury or collagen degradation. 
Fig. 3 Hematoxylin and eosin (H\&E, left panel) and Luxol fast blue (LFB, right panel) staining of longitudinal sections of the right vagus nerve at the electrode site from a representative animal, showing scattered, minimal to mild amounts of neutrophils in the epineurium, perineurium, and nerve
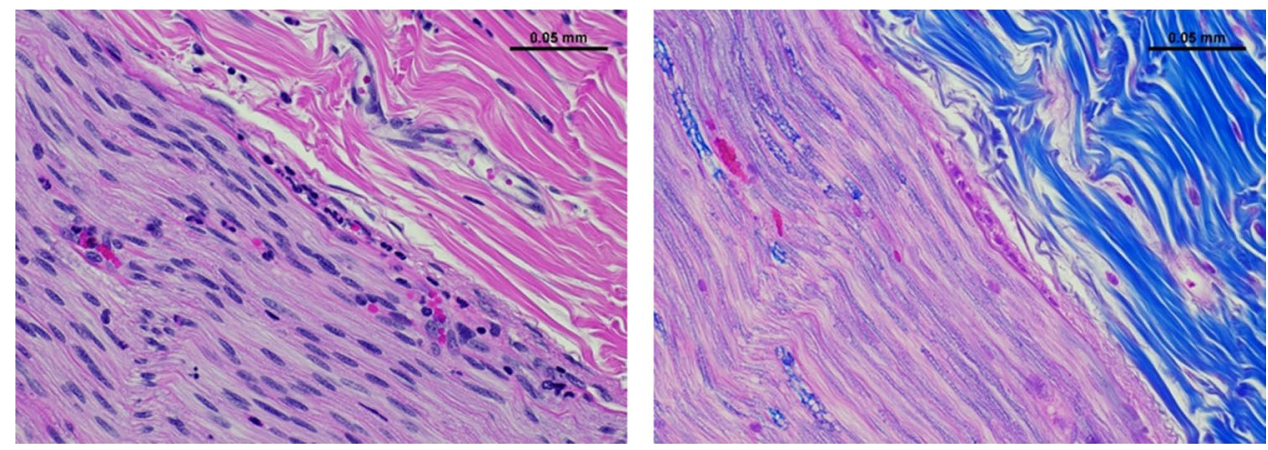

There was no difference between histopathologic parameters in samples taken from the left and right nerve.

\section{Discussion}

ART is a novel HF therapy that was shown in the ANTHEMHF study to significantly improve cardiac function and reduce heart failure symptoms in patients with $\mathrm{HF}$ and reduced EF [4, 5, 18]. Preclinical studies suggest that the beneficial effects of ART derive from multiple synergistic mechanisms in which chronic ART improves regulatory control of the autonomic nervous system. VNS inhibits neural release of norepinephrine at cardiac effectors [19], restores autonomic balance as reflected in improvements in heart rate variability and baroreflex sensitivity [20, 21], reduces systemic inflammation [22-24], increases coronary flow [25], is anti-apoptotic [20, 26], directly modulates reflex processing within peripheral ganglia of the cardiac nervous system [27], and has anti-arrhythmic effects [21, 28-30, 32, 32, 33].

Because patients with HF and reduced EF are at risk for life-threatening ventricular arrhythmias, these patients commonly receive implantable defibrillator therapy (either ICD or S-ICD) and external defibrillation. As these patients may also receive neurostimulation for $\mathrm{HF}$ using an implantable system, it has been important to determine that such systems are able to survive the application of DF energy and continue to perform as originally programmed. Previous benchtop testing according to international testing standards established that implantable ART systems are unaffected by high-energy defibrillation in vitro [13].

In this in vivo study, the VITARIA ART system was evaluated after repeated defibrillation shocks were applied to electrodes in and on the thorax using ICD, S-ICD, and ECD systems. In each configuration, three sequential shocks were delivered, followed by three sequential reverse-polarity shocks. System programming and comprehensive electrical testing confirmed that the implanted ART system was unaffected by the applied energy, and continued to function normally throughout and after the procedure. Analysis of autonomic engagement and nerve histology showed that the vagus nerve was not damaged by defibrillation.

In addition to evaluating the effect that defibrillation has on the ART system, it is also important to evaluate the effect that ART stimulation has on cardiac sensing of ICDs, cardiac pacemakers, and cardiac resynchronization therapy (CRT) devices. This testing was previously performed and published [34], and demonstrated that maximum ART stimulation intensity did not result in a detectable level of interference with either bipolar and unipolar sensing.

\section{Conclusions}

An implantable ART system was tested in vivo for immunity to defibrillation via ICD, S-ICD, and ECD, and was found to be unaffected by a series of high-energy defibrillation shocks. The study results confirm that ART systems are capable of continuing to function appropriately after defibrillation and the cervical vagus nerve is unaffected.

\section{Declarations}

Conflict of interest Dr. Libbus, Mr. Stubbs, and Drs. KenKnight and DiCarlo are employees and shareholders of LivaNova. Mr. Mazar and Mr. Mindrebo are consultants to LivaNova.

Open Access This article is licensed under a Creative Commons Attribution 4.0 International License, which permits use, sharing, adaptation, distribution and reproduction in any medium or format, as long as you give appropriate credit to the original author(s) and the source, provide a link to the Creative Commons licence, and indicate if changes were made. The images or other third party material in this article are included in the article's Creative Commons licence, unless indicated otherwise in a credit line to the material. If material is not included in the article's Creative Commons licence and your intended use is not permitted by statutory regulation or exceeds the permitted use, you will need to obtain permission directly from the copyright holder. To view a copy of this licence, visit http://creativecommons.org/licenses/by/4.0/. 


\section{References}

1. Borovac J, D'Amario D, Bozic J, Glavas D. Sympathetic nervous system activation and heart failure: current state of evidence and the pathophysiology in the light of novel biomarkers. World J Cardiol. 2020;12:373-408.

2. Anand I, Konstam M, Ardell JL, Libbus I and Dicarlo L. Neuromodulation for drug-refractory epilepsy and chronic heart failure: targets, delivery, composition and titration. Int J Neurol Neurother. 2019;6.https://doi.org/10.23937/2378-3001/14100 91.

3. Anand IS, Libbus I, DiCarlo LA. Long-term lead performance for vagus nerve stimulation: low rate of complications and failures. NeuroRegulation. 2020;7:26-9.

4. Premchand RK, Sharma K, Mittal S, Monteiro R, Dixit S, Libbus I, DiCarlo LA, Ardell JL, Rector TS, Amurthur B, KenKnight BH, Anand IS. Autonomic regulation therapy via left or right cervical vagus nerve stimulation in patients with chronic heart failure: results of the ANTHEM-HF trial. J Card Fail. 2014;20:808-16.

5. Premchand RK, Sharma K, Mittal S, Monteiro R, Dixit S, Libbus I, DiCarlo LA, Ardell JL, Rector TS, Amurthur B, KenKnight BH, Anand IS. Extended follow-up of patients with heart failure receiving autonomic regulation therapy in the ANTHEM-HF study. J Card Fail. 2016;22:639-42.

6. Premchand RK, Sharma K, Mittal S, Monteiro R, Dixit S, Libbus I. Long-term follow-up of reduced ejection fraction heart failure patients receiving autonomic regulation therapy in the ANTHEM-HF pilot study. J Am Coll Cardiol. 2019;73:770.

7. Konstam MA, Udelson JE, Butler J, Klein HU, Parker JD, Teerlink JR, Wedge PM, Saville BR, Ardell JL, Libbus I and DiCarlo LA. Impact of autonomic regulation therapy in patients with heart failure: ANTHEM-HFrEF pivotal study design. Circ Heart Fail. 2019;12:e005879.

8. ACC/AHA/HFSA Focused Update on New Pharmacological Therapy for Heart Failure: an update of the. ACCF/AHA Guideline for the Management of Heart Failure: a report of the American College of Cardiology/American Heart Association Task Force on Clinical Practice Guidelines and the Heart Failure Society of America. J Card Fail. 2013;2016(22):659-69.

9. Zipes DP, Camm AJ, Borggrefe M, Buxton AE, Chaitman B, Fromer M, Gregoratos G, Klein G, Moss AJ, Myerburg RJ, Priori SG, Quinones MA, Roden DM, Silka MJ, Tracy C, Blanc JJ, Budaj A, Dean V, Deckers JW, Despres C, Dickstein K, Lekakis J, McGregor K, Metra M, Morais J, Osterspey A, Tamargo JL, Zamorano JL, Smith SC Jr, Jacobs AK, Adams CD, Antman EM, Anderson JL, Hunt SA, Halperin JL, Nishimura R, Ornato JP, Page RL, Riegel B. ACC/AHA/ESC 2006 guidelines for management of patients with ventricular arrhythmias and the prevention of sudden cardiac death-executive summary: a report of the American College of Cardiology/American Heart Association Task Force and the European Society. Eur Heart J. 2006;27:2099-140.

10. Yancy CW, Jessup M, Bozkurt B, Butler J, Casey DE, Colvin MM, Drazner MH, Filippatos GS, Fonarow GC, Givertz MM, Hollenberg SM, Lindenfeld J, Masoudi FA, McBride PE, Peterson PN, Stevenson LW and Westlake C. 2017 ACC/AHA/ HFSA Focused Update of the 2013 ACCF/AHA Guideline for the Management of Heart Failure: a report of the American College of Cardiology/American Heart Association Task Force on Clinical Practice Guidelines and the Heart Failure Society of America. Circulation. 2017.

11. Yancy CW, Jessup M, Bozkurt B, Butler J, Casey DE Jr, Drazner MH, Fonarow GC, Geraci SA, Horwich T, Januzzi JL, Johnson MR, Kasper EK, Levy WC, Masoudi FA, McBride PE, McMurray JJ, Mitchell JE, Peterson PN, Riegel B, Sam F,
Stevenson LW, Tang WH, Tsai EJ, Wilkoff BL. 2013 ACCF/ AHA guideline for the management of heart failure: a report of the American College of Cardiology Foundation/American Heart Association Task Force on practice guidelines. Circulation. 2013;128:e240-327.

12. Implants for Surgery - Active Implantable Medical Devices - Part 1: General Requirements for Safety, Marking and for Information to be Provided by the Manufacturer. 2014;ISO 14708-1.

13. Libbus I, Stubbs SR, Mazar ST, Mindrebo S, KenKnight BH and DiCarlo LA. Effect of defibrillation on the performance of an implantable vagus nerve stimulation system. Bioelectronic Medicine. 2021; In Press.

14. Giordano F, Zicca A, Barba C, Guerrini R, Genitori L. Vagus nerve stimulation: surgical technique of implantation and revision and related morbidity. Epilepsia. 2017;58(Suppl 1):85-90.

15. Kaya E, Rassaf T and Wakili R. Subcutaneous ICD: current standards and future perspective. Int J Cardiol Heart Vasc. 2019;24:100409.

16. Libbus I, Stubbs SR, Mazar ST, Mindrebo S, KenKnight BH, DiCarlo LA. Effect of defibrillation on the performance of an implantable vagus nerve stimulation system. Bioelectronic Medicine. 2021;7:3.

17. Nearing BD, Libbus I, Amurthur B, Kenknight BH, Verrier RL. Acute autonomic engagement assessed by heart rate dynamics during vagus nerve stimulation in patients with heart failure in the ANTHEM-HF Trial. J Cardiovasc Electrophysiol. 2016;27:1072-7.

18. Sharma K, Premchand RK, Mittal S, Monteiro R, Libbus I, DiCarlo LA, Ardell JL, Amurthur B, KenKnight BH and Anand IS. Long-term follow-up of patients with heart failure and reduced ejection fraction receiving autonomic regulation therapy in the ANTHEM-HF pilot study. Int J Cardiol. 2020.

19. Kawada T, Yamazaki T, Akiyama T, Li M, Ariumi H, Mori H, Sunagawa K, Sugimachi M. Vagal stimulation suppresses ischemia-induced myocardial interstitial norepinephrine release. Life Sci. 2006;78:882-7.

20. Zhang Y, Popovic ZB, Bibevski S, Fakhry I, Sica Da, Van Wagoner DR and Mazgalev TN. Chronic vagus nerve stimulation improves autonomic control and attenuates systemic inflammation and heart failure progression in a canine high-rate pacing model. Circulation Heart failure. 2009;2:692-629.

21. Libbus I, Nearing BD, Amurthur B, KenKnight BH, Verrier RL. Autonomic regulation therapy suppresses quantitative T-wave alternans and improves baroreflex sensitivity in patients with heart failure enrolled in the ANTHEM-HF study. Heart Rhythm. 2016;13:721-8.

22. Aukrust P, Gullestad L, Ueland T, Damas JK, Yndestad A. Inflammatory and anti-inflammatory cytokines in chronic heart failure: potential therapeutic implications. Ann Med. 2005;37:74-85.

23. Borovikova LV, Ivanova S, Zhang M, Yang H, Botchkina GI, Watkins LR, Wang H, Abumrad N, Eaton JW, Tracey KJ. Vagus nerve stimulation attenuates the systemic inflammatory response to endotoxin. Nature. 2000;405:458-62.

24. Pavlov VA, Wang H, Czura CJ, Friedman SG, Tracey KJ. The cholinergic anti-inflammatory pathway: a missing link in neuroimmunomodulation. Mol Med. 2003;9:125-34.

25. Henning RJ, Sawmiller DR. Vasoactive intestinal peptide: cardiovascular effects. Cardiovasc Res. 2001;49:27-37.

26. Uemura K, Li M, Tsutsumi T, Yamazaki T, Kawada T, Kamiya A, Inagaki M, Sunagawa K, Sugimachi M. Efferent vagal nerve stimulation induces tissue inhibitor of metalloproteinase- 1 in myocardial ischemia-reperfusion injury in rabbit. Am J Physiol Heart Circ Physiol. 2007;293:H2254-61.

27. McGuirt AS, Schmacht DC, Ardell JL. Autonomic interactions for control of atrial rate are maintained after SA nodal parasympathectomy. Am J Physiol. 1997;272:H2525-33. 
28. Schwartz PJ, Billman GE and Stone HL. Autonomic mechanisms in ventricular fibrillation induced by myocardial ischemia during exercise in dogs with healed myocardial infarction. An experimental preparation for sudden cardiac death. Circulation. 1984;69:790-800.

29. Zuanetti G, De Ferrari GM, Priori SG, Schwartz PJ. Protective effect of vagal stimulation on reperfusion arrhythmias in cats. Circ Res. 1987;61:429-35.

30. Vanoli E, De Ferrari GM, Stramba-Badiale M, Hull SS, Foreman RD, Schwartz PJ. Vagal stimulation and prevention of sudden death in conscious dogs with a healed myocardial infarction. Circ Res. 1991;68:1471-81.

31. Kolman BS, Verrier RL, Lown B. The effect of vagus nerve stimulation upon vulnerability of the canine ventricle: role of sympatheticparasympathetic interactions. Circulation. 1975;52:578-85.

32. Li M, Zheng C, Sato T, Kawada T, Sugimachi M, Sunagawa K. Vagal nerve stimulation markedly improves long-term survival after chronic heart failure in rats. Circulation. 2004;109:120-4.
33. Shinlapawittayatorn K, ChindaDvm K, Palee S, Surinkaew S, Thunsiri K, Weerateerangkul P, Chattipakorn S, Ken Knight BH, Chattipakorn N. Low-amplitude, left vagus nerve stimulation significantly attenuates ventricular dysfunction and infarct size through prevention of mitochondrial dysfunction during acute ischemia-reperfusion injury. Heart Rhythm. 2013;10(11):1700-7.

34. Libbus I, Mazar ST, Stubbs SR, KenKnight BH. Electrical interaction between implantable vagus nerve stimulation device and implantable cardiac rhythm management device. Annu Int Conf IEEE Eng Med Biol Soc. 2018;2018:3681-4.

Publisher's note Springer Nature remains neutral with regard to jurisdictional claims in published maps and institutional affiliations. 